This document is the accepted manuscript version of the following article:

Tumbajoy-Spine1, D., Descartes, S., Bergheau, J. M., Lacai11e, V., Gui11onneau, G., Mich1er, J., \& Kermouche, G. (2016). Assessment of mechanical property gradients after impact-based surface treatment: application to pure $\alpha$-iron. Materials Science and Engineering A: Structural Materials: Properties, Microstructure and Processing, 667, 189-198.

https://doi.org/10.1016/j.msea.2016.04.059

This manuscript version is made available under the CC-BY-NC-ND 4.0 1icense

http://creativecommons.org/1icenses/by-nc-nd/4.0/

\title{
Assessment of mechanical property gradients after impact-based surface treatment: application to pure $\alpha$-iron
}

\author{
David Tumbajoy-Spinel ${ }^{\mathrm{a},{ }^{*}}$, Sylvie Descartes ${ }^{\mathrm{b}}$, Jean-Michel Bergheau ${ }^{\mathrm{c}}$, Victor Lacaille ${ }^{\mathrm{a}, \mathrm{c}, \mathrm{d}}$, Gaylord Guillonneau ${ }^{\mathrm{e}, \mathrm{f}}$, \\ Johann Michlere, Guillaume Kermouche ${ }^{\mathrm{a}}$ \\ ${ }^{a}$ Université de Lyon, Ecole des Mines de Saint-Etienne, CNRS UMR5307, LGF, F-42023 Saint-Etienne, France \\ ${ }^{\mathrm{b}}$ Université de Lyon, INSA-Lyon, CNRS UMR5259, LaMCoS, F-69621 Villeurbanne, France \\ ${ }^{c}$ Université de Lyon, ENISE, CNRS UMR5513, LTDS, F-42023 Saint-Etienne, France \\ d Winoa, 528 Avenue de Savoie, BP3, 38570 Le Cheylas, France \\ ${ }^{\mathrm{e}}$ Empa, Swiss Federal Laboratories for Materials Science and Technology, Laboratory for Mechanics of Materials and Nanostructures, Feuerwerkerstr. 39 , CH-3602 Thun, Switzerland \\ ${ }^{\mathrm{f}}$ Université de Lyon, Ecole Centrale de Lyon, CNRS UMR5513, LTDS, F-69134 Ecully, France
}

\begin{abstract}
Mechanical surface treatments are known for their ability to improve material resistance to abrasive wear and local fatigue crack microstructure of the home-made crack propagation. These treatments are based on repeated contact loadings which create large plastic strains in the near-surface that can induce a local grain refinement. In this case, a significant increase in the near-surface local mechanical properties is thus usually observed. In this paper, nano-mechanical tests are used to quantify the mechanical property gradient in the near-surface of a purity-controlled $\alpha$-iron after an impact-based treatment. A methodology based on the combination of two different techniques is proposed: nano-indentation and in-situ micro-pillar compression. The resulting in-depth mechanical properties gradient is compared to the average grain size measured by EBSD. A positive relationship with the well-known Hall-Petch effect is observed.
\end{abstract}

\section{Introduction}

Surface mechanical treatments are used increasingly to maximize the lifetime of engineering parts by altering the near-surface mechanical properties. These treatments are based on repeated contact loading [1-3]. On the one hand, this can result in the creation of a local residual stress field which can prevent fatigue crack propagation, while on the other hand severe plastic deformation can occur in the near-surface which can lead to local microstructure transformation $[4,5]$. The latter is characterized by a progressive grain size refinement and consequently the formation of a mechanical property gradient over a few tens of microns [6]. The hardening of the near-surface is closely associated with an increase in grain boundary and dislocation density. The resulting ultra-fine grain zone is usually called a tribologically transformed surface (TTS) $[7,8]$. Beneficial physical properties such as high hardness and improved tribological properties are generally exhibited in these mechanically-induced transformed surfaces. The understanding of these surface mechanical treatments requires

\footnotetext{
* Corresponding author.
}

the development of adapted characterization methods to accurately measure micro-structural and local mechanical properties, which is important as strong variation in-depth properties may appear because of microstructural gradients.

Two types of testing approaches for such measurements are nano-indentation and micro-compression [9,10]. While nanoindentation testing appears to be one of the best methods to reach this goal, data analysis remains a non-trivial task due to the well-known problem of contact area determination under load. It is thus difficult to obtain stress-strain curves at the length scale of the surface treatment, although this is of primary interest to engineers, especially for modeling purposes [9]. In contrast, micropillar compression tests could be a more direct method to accurately measure local stress-strain curves and obtain the mechanical property gradients in-depth. Nevertheless, the micro-pillar fabrication process in polycrystalline zones is a heavily laborious task [10], and is inherently difficult to translate to industrial purposes.

The main aim of this paper is to propose a new methodology based on the combination of nano-indentation and in situ micropillar compression for the assessment of micromechanical 
properties. In the past, several authors have independently used both of these techniques to identify the mechanical property gradient induced by surface treatments (SMAT, sliding deformation, shot-peening, etc.) in metallic materials [11-13]. Nano-indentation testing enables an overall measurement of the mechanical property gradient, while micro-pillar compression allows for an accurate quantification of the local stress-strain curves. Combining these two measurements, it is possible to obtain the yield stress as a function of distance to the surface assuming nanohardness as three times the yield stress (for $8 \%$ of plastic strain) as proposed by Tabor [14-16].

The clear advantage of using both techniques is the possibility to compare two different kinds of measurements, allowing for local direct estimations (micro-compression) with a large testing coverage (nano-indentation) throughout the entire transformed region. Moreover, this combined procedure permits the investigation of some metallurgical effects involved in the surface strengthening, and associate them to the mechanisms governing the microstructural transformations. In other words, this work allows for the comprehension of the influence of surface mechanical treatments through quantification of the local mechanical properties.

This combined strategy is applied to a model material (pure iron), previously submitted to a nano-peening ${ }^{\circledR}$ treatment (impact-based treatment). The microstructure of the transformed zone, as well as the statistical analysis of the grain size distribution on the cross-section of the sample is first presented. Next, the methodology discussed above is applied to quantify the mechanical properties of the transformed zone. A relationship between the hardness gradient and the local microstructure is finally established, as well as a comparison between the properties measured by both techniques.

\section{Process and methods: microstructural refinement on pure $\boldsymbol{\alpha}$-iron}

\subsection{Material}

The mechanical surface treatment is carried out on a highpurity $\alpha$-iron produced using the cold crucible melting method. This enables the formation of $\alpha$-iron with less than $15 \mathrm{ppm}$ of carbon. The metallic bar is treated thermo-mechanically (forging, annealing at $650{ }^{\circ} \mathrm{C}$ for $60 \mathrm{~min}$ ) before applying the surface mechanical treatment. The resulting microstructure is shown in Fig. 1. Grains are equiaxed with an average grain size of approximately $250 \mu \mathrm{m}$. No inclusions were observed. The sample surface was then mechanically polished with diamond suspensions of $3 \mu \mathrm{m}$ and $1 \mu \mathrm{m}$.

\subsection{Impact-based surface treatment: Nano-Peening ${ }^{\circledR}$}

Nano-Peening ${ }^{\circledR}$ is a surface mechanical treatment developed by Winoa group (Fig. 2). Steel balls $(0.1-2 \mathrm{~mm}$ diameter) are repeatedly projected towards the sample surface with an impact tilt between $10^{\circ}$ and $45^{\circ}$ at high speeds (40-100 m/s) [17;18]. Here, a surface coverage of $5000 \%$ has been used. This cold-working treatment is known to lead to severe plastic deformation of the near-surface and thus to grain refinement.

\subsection{Microstructural characterization}

Microstructural characterization was performed on material cross-sections using back-scattering electron microscopy in a Zeiss Supra $55 \mathrm{VP}$ at $20 \mathrm{kV}$. Electron back-scattered diffraction (EBSD) mapping was obtained using a JEOL JSM-6500F at $20 \mathrm{kV}$ and an

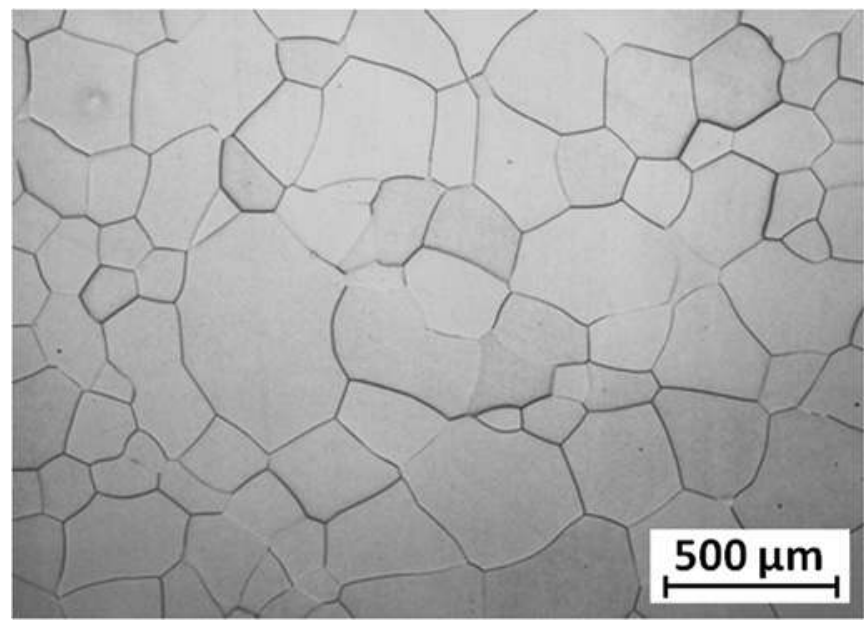

Fig. 1. Initial microstructure of the home-made purity-controlled $\alpha$-iron (15 ppm of carbon). Grains are homogeneous and equiaxed with an average grain size of $\sim 250 \mu \mathrm{m}$.

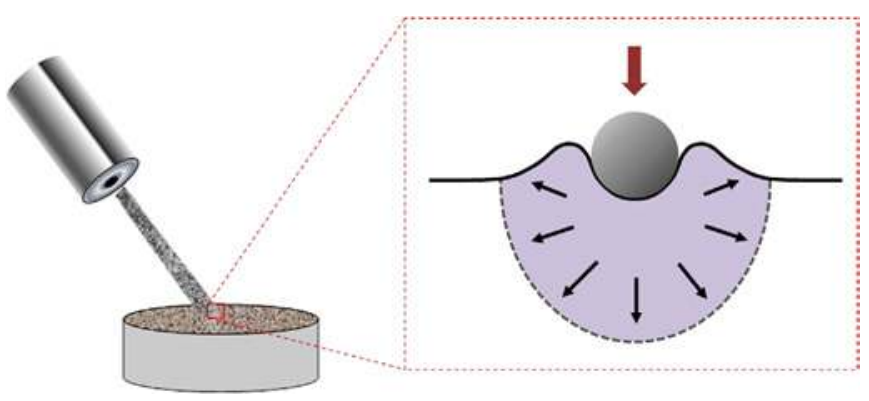

Fig. 2. Impact-based surface treatment: Nano-Peening ${ }^{\mathbb{R}}$.

indexation step size of $0.3 \mu \mathrm{m}$. Note that this step size was chosen to limit indexation drifts and have a reasonable acquisition time. Cross-sections were prepared using mechanical polishing until a diamond suspension size of $1 \mu \mathrm{m}$. Surface finishing was carried out using colloidal silica.

Three different regions can be clearly identified in the treated surface (Fig. 3a and c). The first zone corresponds to a tribologically transformed surface (TTS) composed of well-defined submicrometric grains (Fig. $3 \mathrm{~b}$ and $\mathrm{d}$ ). The thickness of this zone varies from $30 \mu \mathrm{m}$ to $80 \mu \mathrm{m}$ with an average value of $50 \mu \mathrm{m}$. The TTS region is composed of grains with sizes predominately below $1 \mu \mathrm{m}$ diameter with various crystal orientations. This refined microstructure is followed by a transition zone, where no new grains are formed in spite of the severe deformation and crystal misorientation [19]. Finally the third zone corresponds to the initial bulk material without microstructural transformation (as shown in Fig. 1).

\subsection{Measurement of mechanical properties by nano-mechanical testing}

The grain size decrease and the microstructural gradient are closely related to the change in mechanical properties from the near-surface to the inner bulk material. Two different nano-mechanical testing techniques are employed to quantify this gradual variation on the mechanical behavior: nano-indentation and micro-compression (Fig. 4). Nano-indentation permits the estimation of the hardness $(\mathrm{H})$ and Young's modulus (E) from the maximal load point of the indentation curve (as outlined in Fig. 4). In contrast, the micro-pillar compression test leads to the measurement of true stress-strain behavior, discounting for 

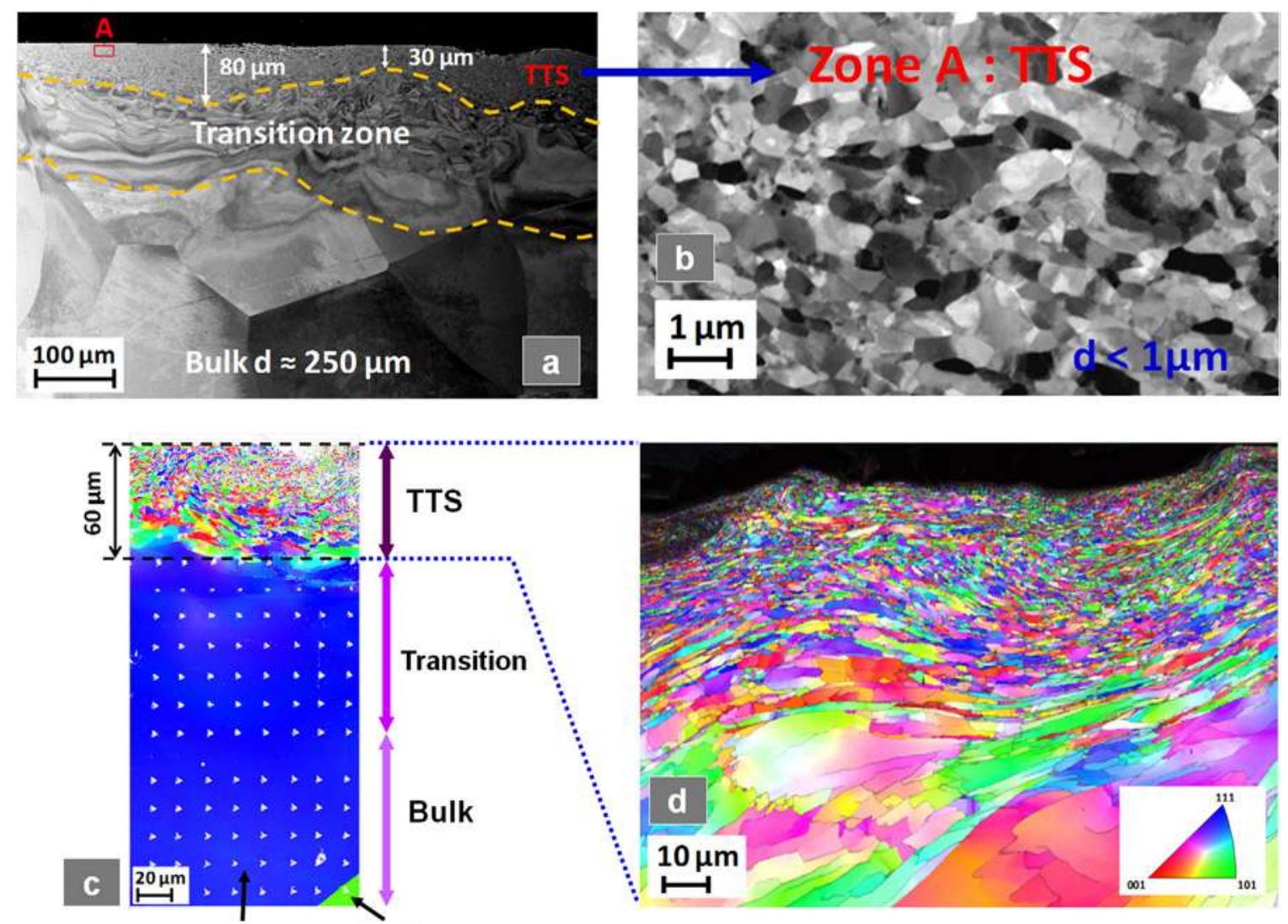

(111)

(110)

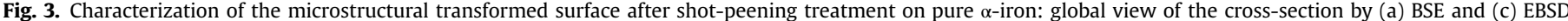
map; local view by (b) BSE and (d) EBSD map. Note that the small white spots in figure (c) correspond to nano-indentation prints.
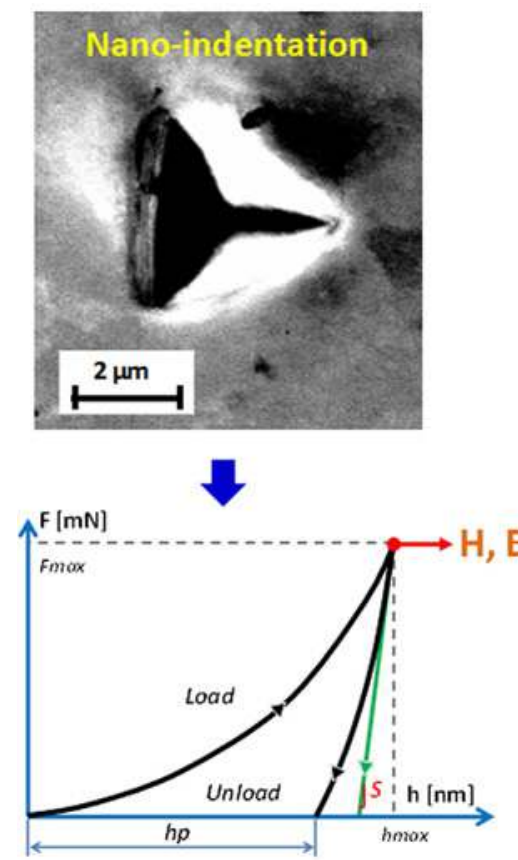
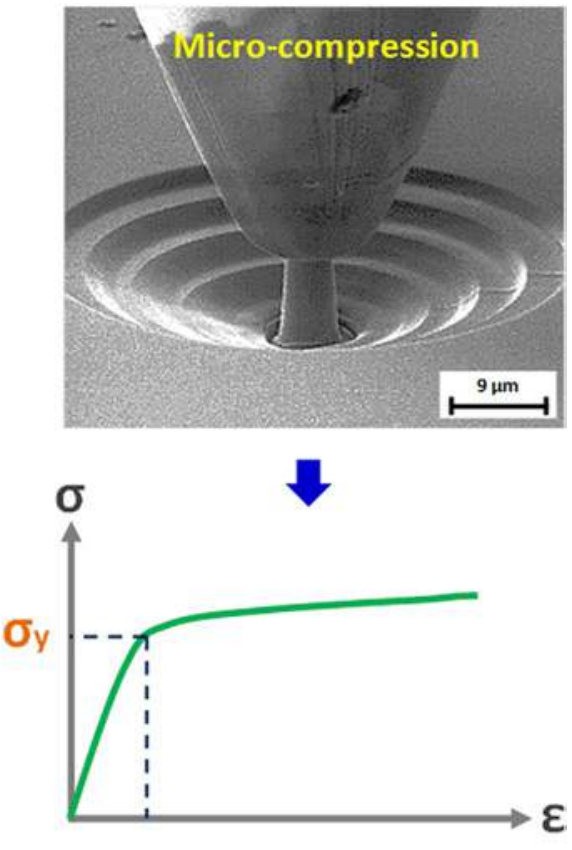

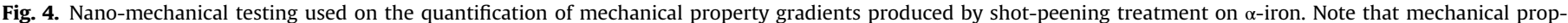
erties, hardness $(\mathrm{H})$ and elastic modulus $(\mathrm{E})$, are obtained from nano-indentation testing at the maximal load point of the force-displacement curve.

strains lower than a few percent where uncertainties in compression direction and the pillar surface roughness make precise measurement of elastic properties difficult.

\subsubsection{Nano-indentation testing}

Nanoindentation tests have been performed with an Anton Paar SA ${ }^{\mathbb{R}}$ NHT nanoindenter using a Berkovich tip. Tests were arranged in a rectangular grid matrix $(10 \times 20)$, with a $15 \mu \mathrm{m}$ spacing 
between indents on both vertical and horizontal axes (Fig. 5a). Twenty different depths were investigated. A maximal force of $10 \mathrm{mN}$ is applied with a load-unload rate of $20 \mathrm{mN} / \mathrm{min}$. Hardness and elastic moduli are computed using the contact stiffness $[20,22]$, which is measured as the initial slope of the unloading part of the force-displacement curve (Fig. 4) [23,24] using the Loubet's contact model [21]. Note that the distance between indentations $(15 \mu \mathrm{m})$ is higher than three times the characteristic length of the residual imprints $(\leq 4 \mu \mathrm{m})$ to avoid perturbations due to the mechanically affected zone of adjacent tests (Fig. 5b and c) [25]. An indentation force of $10 \mathrm{mN}$ permits the investigation of several positions in the TTS region without interfering with adjacent measurements. For comparative purposes, the same indentation force has been used throughout the whole cross-section.

\subsubsection{Micro-pillar compression}

As for nano-indentation tests, micro-pillar compressions are performed over the cross-section. Two pillars are produced in the TTS and bulk regions as shown in Fig. 6a. These were milled using a gallium source of the Zeiss NVision40 focused ion beam (FIB) system. The fabrication process includes 3 rough milling phases and a final polishing step [26-28]. The gallium beam is used at $30 \mathrm{kV}$ and the milling current ranges from $27 \mathrm{nA}$ for rough milling to $700 \mathrm{pA}$ for final polishing. This strategy allows for cleaner and more homogenous pillar surfaces to be obtained. The aspect ratios of the TTS and bulk pillars (Fig. 6b and c) are 2.6 (9.3 $\mu \mathrm{m}$ diameter and $24 \mu \mathrm{m}$ height) and 1.9 (5.4 $\mu \mathrm{m}$ diameter and $10.4 \mu \mathrm{m}$ height) respectively. The TTS pillar is located at a depth of $15 \mu \mathrm{m}$ from the surface, which corresponds to a polycrystalline material of ca. $800 \mathrm{~nm}$ average grain size. The polycrystalline pillar contains approximately 11 grains across the diameter. The bulk pillar was produced in a grain with crystal orientation $\langle 225\rangle$ with respect to the load direction. Both pillars have a conical shape with a tapered angle of $2.5^{\circ}$ and $4.5^{\circ}$ for the TTS and bulk zones respectively. The compression tests were made in situ in a scanning electron microscope (SEM) with an Alemnis nano-indentation set-up [29]. Both compressions were carried out with a $15 \mu \mathrm{m}$ diameter flat punch at a load-unload rate of $0.03 \mu \mathrm{m} / \mathrm{s}$.

\section{Results}

\subsection{Grain size distribution of the mechanically transformed surface}

The average grain size was measured in the mechanically transformed surface from the EBSD map shown in Fig. 3c. It evolves from $0.76 \mu \mathrm{m}$ up to $5.82 \mu \mathrm{m}$ at the bottom limit of the TTS region, i.e. roughly $60 \mu \mathrm{m}$ from the surface (Fig. 7). It is important to note that the ratio between the grain size at the near-surface and the initial bulk grain dimensions is approximately 330 .

Concerning the transition zone, the EBSD map as well as the SEM image (Fig. 3a and c) highlight the presence of considerable crystal misorientations produced by severe plastic deformation. However, the deformation level is not severe enough to promote the creation of new grain boundaries [19].

\subsection{Hardness gradient measured by nano-indentation testing}

Arrays of residual imprints at two different depths are presented in Fig. 5b and c. The lateral dimensions are closely related to the microstructural evolution along the cross-section, highlighting the increase in mechanical properties for the near-surface.
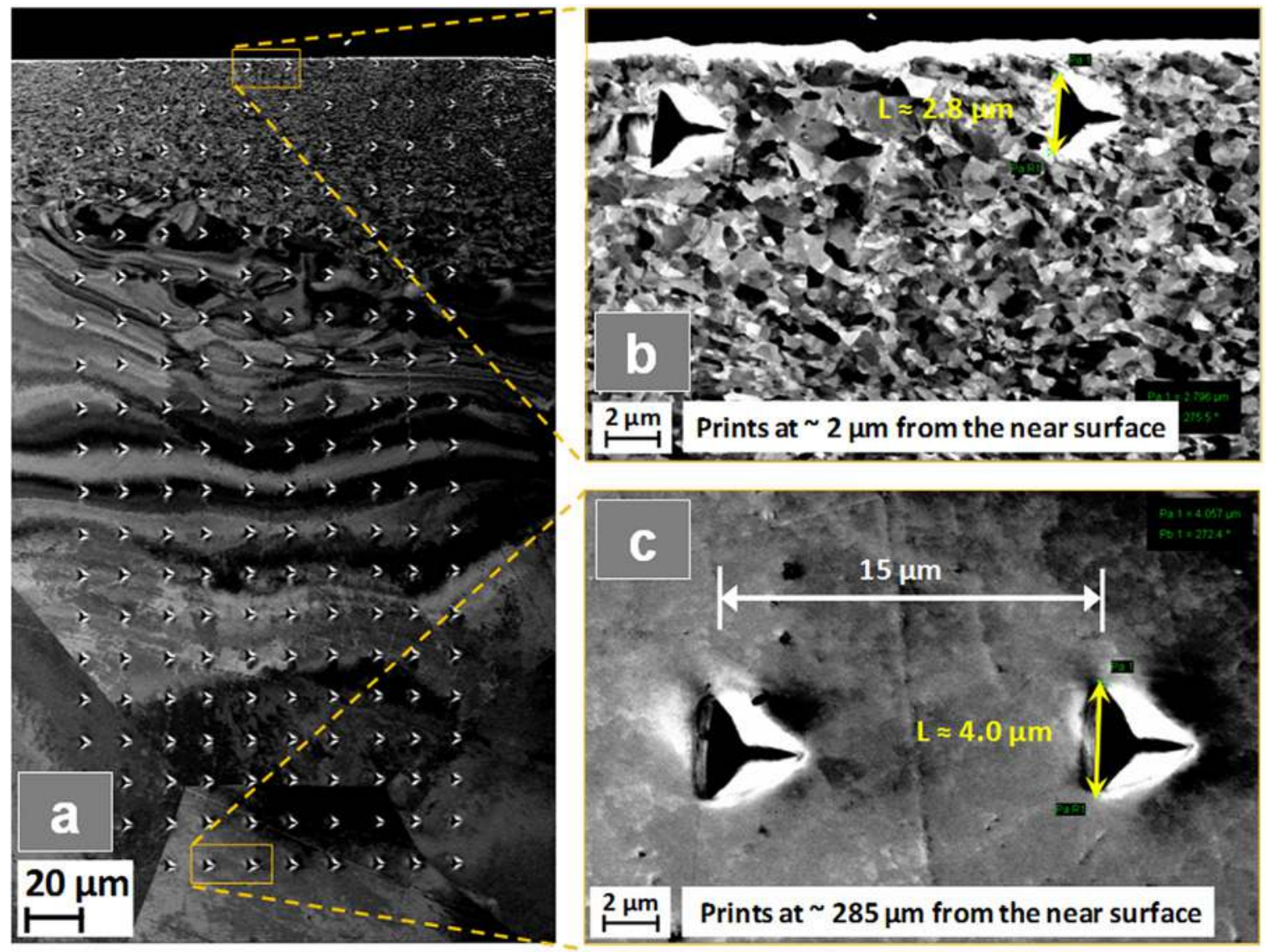

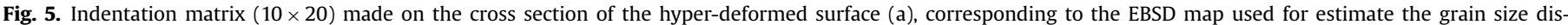
tribution. Indentations prints with $15 \mu \mathrm{m}$ spacing and a force of $10 \mathrm{mN}$ with a Berkovich tip (b, c). 


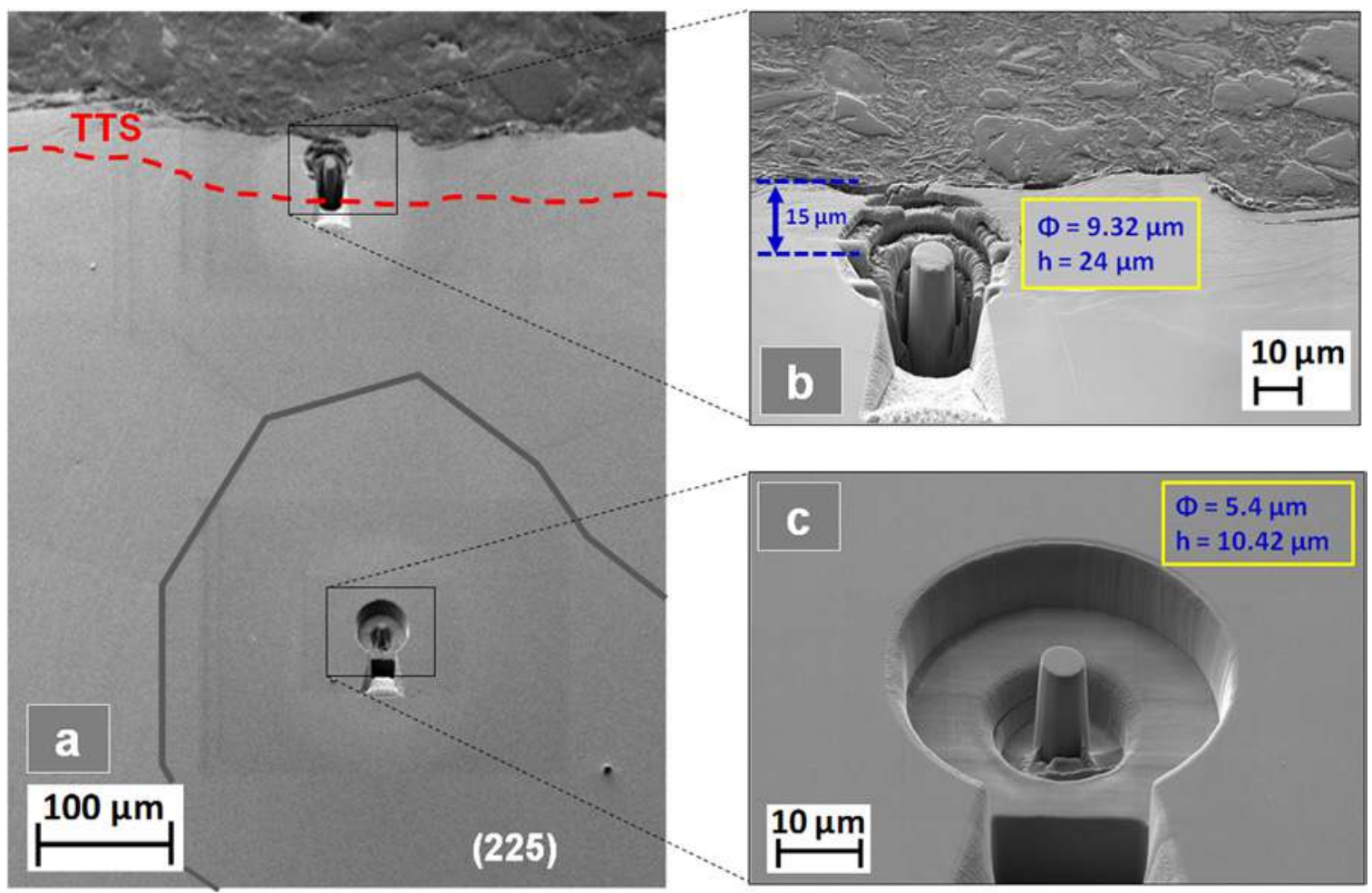

Fig. 6. (a) Micro-pillars position on the cross-section, (b) TTS micro-pillar and (c) bulk micro-pillar.

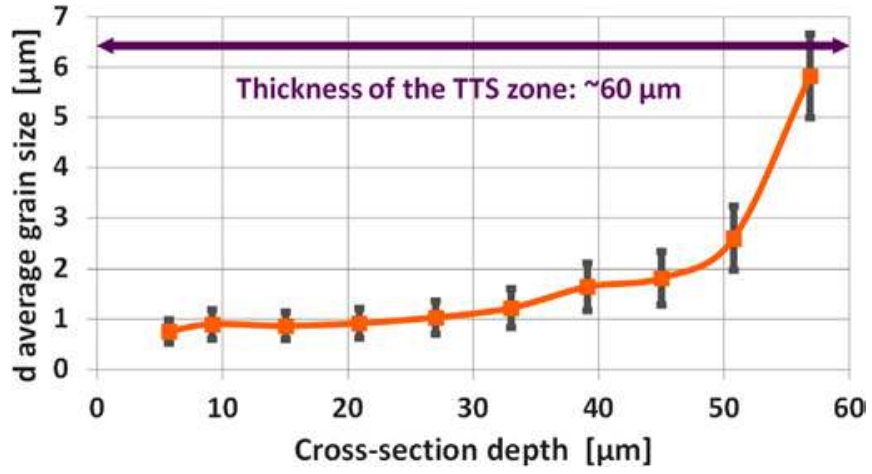

Fig. 7. Grain sizes distribution as a function of the cross-section depth in the TTS zone.

For instance, the characteristic size of the residual impression at ca. $2 \mu \mathrm{m}$ depth is approximately $2.8 \mu \mathrm{m}$, contrary to the one located at ca. $285 \mu \mathrm{m}$ from the surface where the length increases up to $4 \mu \mathrm{m}$. According to the EBSD map in Fig. 3c, the nano-indentation tests in the bulk material were predominately carried out for a $\langle 111\rangle$ crystal surface orientation.

The in-depth hardness and Young's modulus are plotted in Fig. $8 \mathrm{a}$ and $\mathrm{b}$. In both curves, each point corresponds to the average value obtained from the ten indentation tests carried out at each depth. The measurements performed at $2 \mu \mathrm{m}$ from the surface, corresponding to the first point of each curve, are mostly erroneous, and as such have not been taken into account. The average hardness decreases from $2300 \mathrm{MPa}$ in the near-surface, to $1250 \mathrm{MPa}$ at $150 \mu \mathrm{m}$ depth (Fig. 8a). The hardness is therefore reduced by $40 \%$, of which $30 \%$ corresponds to the first $60 \mu \mathrm{m}$ of the TTS layer, followed by a $10 \%$ reduction over the transition zone. The plateau corresponds to the initial bulk material properties.

As expected, Young's modulus remains constant at ca. $207 \mathrm{GPa}$ $[23,30]$ over the entire cross-section. The elastic modulus represents the material atomic binding forces responding to an external load
[30], while several investigations have pointed out that the Young's modulus could vary in a material due to different reasons: temperature variations [31], material porosity [30], phase transformations [31] or crystallographic orientations. In this work, the homogeneous features of high-purity $\alpha$-iron as well as the multidimensional loading condition of indentation tests [32] could explain this low elastic modulus anisotropy, even in the presence of a significant grain size refinement and various crystal orientations.

\subsection{Micro-compression test results}

The results of in situ compression tests are presented in Fig. 9. It was observed that the TTS pillar deformation is entirely homogeneous (Fig. 9a-c), which is expected due to its polycrystalline nature. On the other hand, the pillar situated in the bulk of the sample deforms in a well-defined slip plane, as indicated by the white arrows in Fig. 9e and f. This kind of behavioral feature is typical of single crystals, due to the activation of a preferential slip system under mechanical loads [33].

True stress-strain curves are obtained from the force-displacement data of both micro-compression tests (Fig. 10). Compliance effects from the frame, substrate and indenter were subtracted from the measured force-displacement values during data processing. The yield strength estimated for the TTS and bulk regions were ca. $700 \mathrm{MPa}$ and ca. $300 \mathrm{MPa}$ respectively. Plastic flow was observed through an absence of strain hardening for the TTS pillar, whereas a slight strain-hardening was detected for the single-crystal pillar. Note that the initial yield strength is close to the yield stress at a strain of $8 \%\left(\sigma_{0.08}\right)$, the representative strain related to Berkovich indentation [16,34], for both regions (TTS and bulk). It should be noted that micro-compression does not allow the measurement of initial yield stress with a very good accuracy for metals. Indeed, a very small difference between the compression direction and the pillar axis may prevent an accurate measurement of the elastic component for such materials. As an example, it can be seen that the Young's modulus which could be 
measured from Fig. 10 would be significantly lower than the true Young's modulus of pure iron.

\section{Discussion}

\subsection{Nano-indentation vs micro-compression}

According to the curves presented in Figs. 8 and 10, high increments of mechanical properties were quantified using both
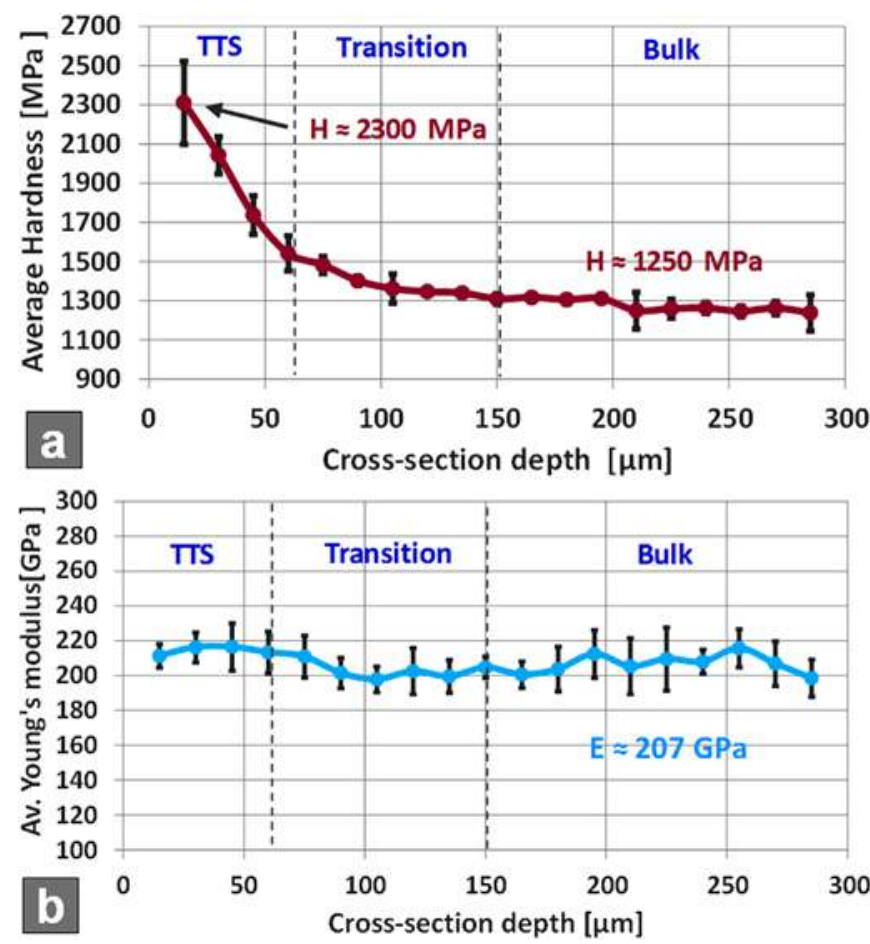

Fig. 8. Evolution of (a) average hardness [MPa] and (b) average Young's modulus [GPa] over the cross-section. Curves are estimated using the model of Loubet. techniques. Micro-compression tests show that the yield strength of the TTS zone is approximately twice that measured in the bulk material region, which is closely related to the hardness gradient estimation (ca. 40\%).

Furthermore, nano-indentation and micro-compression results can be compared within the context of the experimental Tabor relationship for metals $[14,15]$, in which the hardness corresponds to approximately three times the yield strength for a representative strain of $0.08\left(H / \sigma_{0.08} \approx 3\right)$ [16]. The polycrystalline zone presents a ratio of ca. $3.3(2300 \mathrm{MPa} / 700 \mathrm{MPa})$, meanwhile the bulk material region exhibits a ratio of ca. 4.2 (1250 MPa/ $300 \mathrm{MPa}$ ). The latter value deviates slightly from the expected ratio.

In a first approach, one can presume that the discrepancy between the measurements done by nano-mechanical testing in single crystal regions may be related to the different crystal orientations. More precisely, nano-indentation and micro-compression tests in the bulk material were carried out in $\langle 111\rangle$ and $\langle 225\rangle$ orientations, respectively. Considering the 48 possible slip systems for $\alpha$-iron (three kinds of plane and direction families for bcc crystal structures) [35], the maximal Schmid factors with respect to the normal direction of the surface for these two grains are $m_{\langle 111\rangle}=0.314$ and $m_{\langle 225\rangle}=0.433$. Indeed, the indented bulk zone seems to be 1.38 times $\left(m_{\langle 225\rangle} / m_{\langle 111\rangle}\right)$ stronger than the compressed bulk zone, which appears to be consistent with the results above. However, this interpretation should be taken with caution as the multiaxial nature of nano-indentation loading in single crystals could activate several slip systems simultaneously [32].

As was previously presented by Vlassack and Nix [36], the hardness and elastic moduli of single crystals do not vary significantly with respect to the indentation orientation axis. In that work, the anisotropic behavior of single crystals with different orientations ( $\{100\} ;\{110\} ;\{111\})$ was presented for several cubic structure materials: copper, $\beta$-brass, aluminum and tungsten. From numerical simulations and experimental nano-indentation tests, they observed slight anisotropic variation in hardness (less than 13\%) and indentation modulus (between 10\% and 25\%). It would suggest that multiple slip systems are activated during indentation [32,37] and the hardness measurement corresponds to the averaged behavior of all these glide systems. In other words,
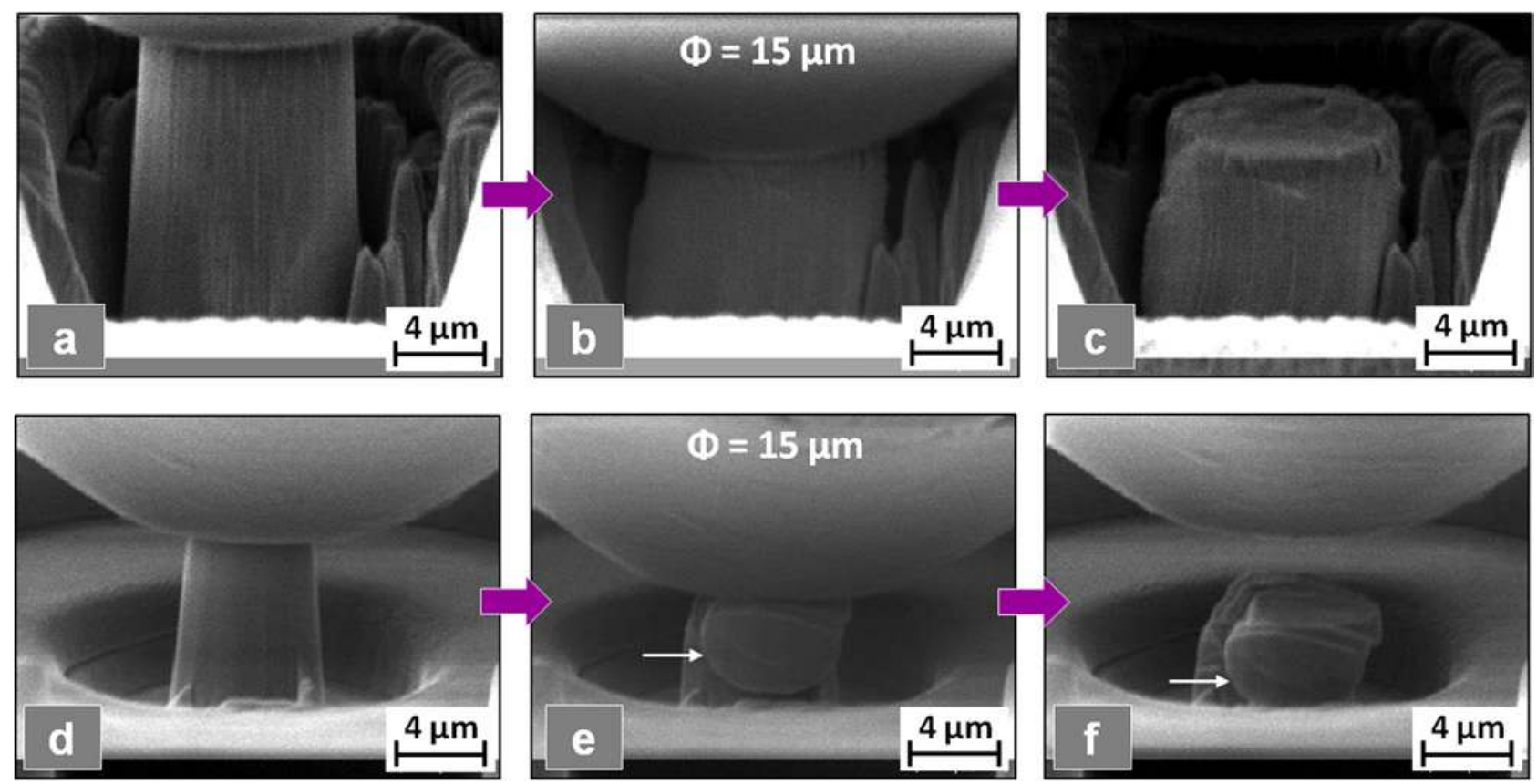

Fig. 9. Micro-pillar compression tests of the TTS region (a,b,c) and the bulk material (d,e,f), using a flat tip of $\Phi 15 \mu \mathrm{m}$. 


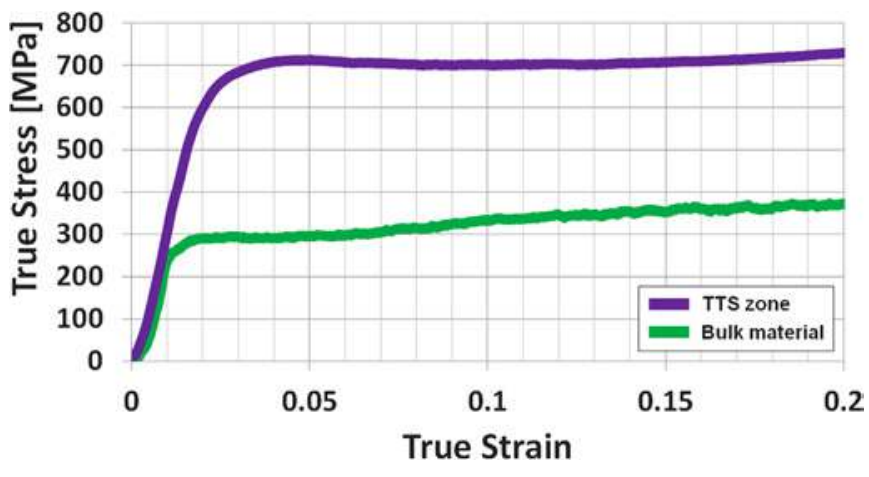

Fig. 10. True stress-strain curves of the TTS zone and bulk micro-pillars.

the hardness does not change so much with the crystal orientation in spite of the significant yield stress anisotropy that could exist in the material [36].

According to that, the difference between the mechanical properties quantified in single crystals is particularly related to the nature of the load and the corresponding plastic deformation effected. Indeed, it could be possible that hardness measurement values in single crystals are higher than those expected on the yield stress basis [36]. Due to the low anisotropy observed from nano-indentation testing, it would not be surprising if the hardness measurement in a $\langle 225\rangle$ indentation direction (same orientation as compression test) remains at ca. $1250 \mathrm{MPa}$. This is clearly visible from hardness measurements corresponding to the last two lines of indentation imprints in the rectangular array shown in Fig. 5-a. As shown in this figure, indentations were performed in other single crystal orientations (for example $\langle 110\rangle$ from the EBSD map in Fig. 3c) and the average hardness remains at approximately $1250 \mathrm{MPa}$ (Fig. 8). Despite the mechanical property differences observed for single crystals in this work, it is noted that the Schmid factor remains a main criterion regarding the comparison between uni-axial load tests. Likewise, nano-mechanical tests were carried out near the transformed region and the influence of dislocation density, which has not been explicitly taken into account in this investigation, could also play a role.

Moreover, micro-compression tests reveal negligible strain hardening for the polycrystalline pillar, in contrast to the bulk material pillar behavior (Fig. 10). Due to the sub-micrometric grain sizes and the severe plastic deformation already introduced in the TTS zone, the pillar deformation could be mostly governed by grain boundary interactions [38] instead of dislocation interactions in the grains (Taylor's expression) [39]. Size effects are not taken into account as both pillars do not have sub-micrometric dimensions; a condition that is specified in literature [40-42].

\subsection{Hardness and grain size relationship: the Hall-Petch effect}

The micro-structural transformation of pure $\alpha$-iron is closely related to its high stacking fault energy, allowing for favorable dislocation movement and migration [43]. In this material, grain refinement occurs essentially in three steps during severe plastic deformation [44,45]. Firstly, for low strain levels, dislocation glide exists in multiple slip planes and the material is arranged in cellular substructures subdivided by high-dislocation-density walls. In this first phase, low angle boundaries are created in the initial grains. Secondly, when the deformation increases up to middle strains, the dislocation sub-boundaries of these cell blocks become considerably misoriented. In this case, multiple micro-shear bands start appearing into the grains with various directions. Several micro-shear band interactions lead to high-angle boundaries dividing the original grains into small structural elements. Finally, there is a saturation of micro-shear bands and dislocation

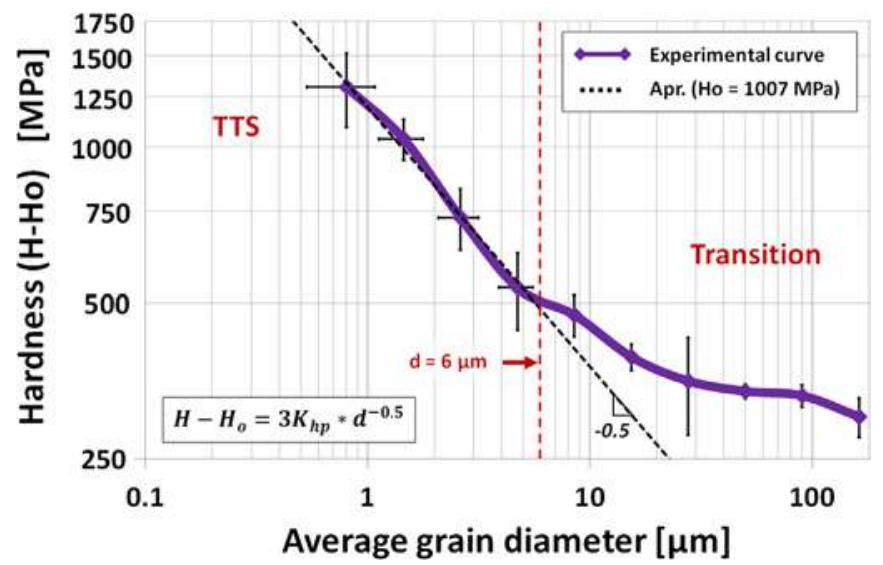

Fig. 11. Power law describing the relationship between the relative mean hardness $(\mathrm{H}-\mathrm{Ho})$ and the average grain diameter $(\mathrm{d})$ on the micro-structured zone: HallPetch effect.

densities allowing for the formation of well-defined grains.

Considering the mechanisms governing this microstructural transformation, it is not surprising that the increase in mechanical properties is closely related to the increase in grain boundaries as well as the rise of local dislocation densities. Nano-indentation testing revealed that the hardness decreases approximately $40 \%$ over a $150 \mu \mathrm{m}$ depth, corresponding to the TTS and transition zones along the cross-section. It is therefore possible to correlate the quantified average hardness gradient with the grain size distribution obtained from the EBSD map (Fig. 11).

Fig. 11 shows that the hardness decreases linearly with the logarithm of the average grain size, which is consistent with microstructure refinement effects, such as the Hall-Petch effect. Once the average grain size goes beyond $6 \mu \mathrm{m}$, i.e. at $60 \mu \mathrm{m}$ depth, the transition zone is reached and the linear tendency vanishes progressively. The grain size in the transition zone corresponds to an extrapolation of the grain size distribution in the TTS layer. As was mentioned in Section 2.3, grains along the transition zone are not well-defined and consequently the Hall-Petch relationship for that region can no longer be applied. As such, the decrease in hardness is likely related to the gradient of dislocation densities and the local misorientation $[4,46]$, also associated with the presence of sub-grain boundaries.

In order to understand the Hall-Petch effect in the sub-micrometric region, the experimental curve in Fig. 11 is fit using the equation: $H-H_{o}=3 K_{h p} d^{-0.5}$, where $K_{h p}$ is a material constant, $H_{o}$ is the bulk material hardness, and $d$ is the grain diameter [47]. The obtained approximation (black dashed line in Fig. 11) corresponds to the following material constants: $H_{0-A p r x}=1007.8 \mathrm{MPa}$ and $K_{h p-A p r x}=397.15 \mathrm{MPa} \mu \mathrm{m}^{0.5}$..

The same experimental values are plot in Fig. 12 to compare the fitted approximation (black dashed line) with the linear Hall-Petch model proposed in literature for $\alpha$-iron (blue line). Both straight lines represent the equation $H=H_{o}+3 K_{h p} d^{-0.5}$, where $H_{o}$ is the intercept of the vertical axis and $3 K_{h p}$ is the slope of the line. The material constants used from literature (blue line) are $K_{h p-F / Z}=480 \mathrm{MPa} \mu \mathrm{m}^{0.5}$ and $H_{0-F / Z}=300 \mathrm{MPa}$, taken from Fu et al. [48] and Zhao et al. [49]. These authors suggest that the $K_{h p}$ value could vary in pure $\alpha$-iron for different grain size ranges [48,49]. For micrometric grain diameters, the Hall-Petch effect is described by a typical linear relationship with a slope of $480 \mathrm{MPa}^{0.5}$ [48]. When the average grain size approaches the nanocrystalline range $(d<100 \mathrm{~nm})$, this linear tendency starts to disappear and the slope evolves continuously to $210 \mathrm{MPa}^{0} \mathrm{~m}^{0.5}$ [48]. Considering the grain size distribution of the TTS zone, the estimated material constant $K_{h p-A p r x}\left(400 \mathrm{MPa}_{\mu} \mathrm{m}^{0.5}\right)$ is in reasonable agreement with 


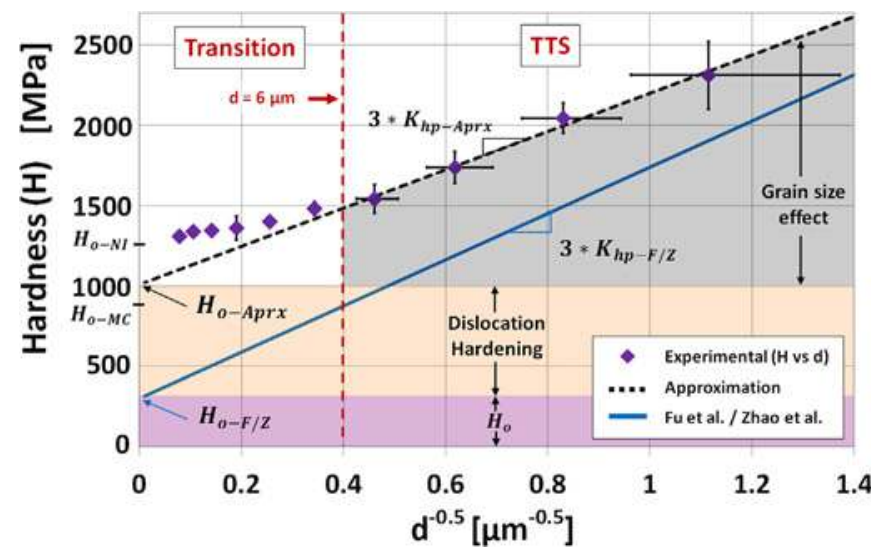

Fig. 12. Comparison of the Hall-Petch effect identified from indentations tests (black dashed line) with models proposed by Fu et al. [48] and Zhao et al. [49] for pure $\alpha$-iron (blue continuous line). Straight lines represent the equation $\boldsymbol{H}=\boldsymbol{H}_{\boldsymbol{o}}+3 \boldsymbol{K}_{\boldsymbol{h} p} \boldsymbol{d}^{-0.5}$, where $\boldsymbol{H}_{\boldsymbol{o}}$ is the intercept of the vertical axis and $3 \boldsymbol{K}_{\boldsymbol{h} p}$ is the slope of the line. The Hall-Petch constants are: $\boldsymbol{H}_{\boldsymbol{o}-\boldsymbol{A p r x}}=1007.8 \mathrm{MPa}$; $\boldsymbol{K}_{\boldsymbol{h} \boldsymbol{p}-\boldsymbol{A p r \boldsymbol { x }}}=397.15 \mathrm{MPa} \mu \mathrm{m}^{0.5}$ for the approximation and $\boldsymbol{H}_{\boldsymbol{o}-\boldsymbol{F} / \boldsymbol{Z}}=300 \mathrm{MPa}$,

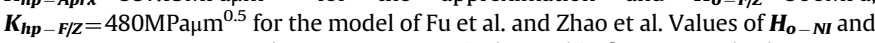
$\boldsymbol{H}_{\boldsymbol{o}-\boldsymbol{M C}}$ are $1250 \mathrm{MPa}$ and $900 \mathrm{MPa}$ respectively. In this figure, purple dots correspond to the experimental data. Note that error bars are not visible in four dots of the transition zone due to the low standard deviation of these points (less than $27 \mathrm{MPa}$ ). (For interpretation of the references to color in this figure legend, the reader is referred to the web version of this article.)

the value for the slope proposed in literature for micrometric

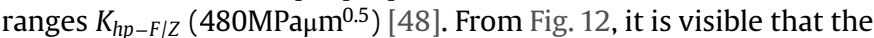
slopes of both straight lines are similar. This could suggest that the grain size effect quantified by nano-indentation tests in the TTS region is consistent with models already proposed in the literature for $\alpha$-iron. The discrepancy between these values is likely caused by a slope drop in the presence of a sub-micrometric grain range, as the indexation step size of the EBSD map $(0.3 \mu \mathrm{m})$ is not enough to reveal grains smaller than $500 \mathrm{~nm}$ in diameter at the nearsurface.

The significant difference between both curves (Fig. 12) is the $y$-axis intercept of each straight line, i.e. the $H_{o}$ value. This value represents the mechanical behavior associated with dislocation interactions and movements during plastic deformation. Some authors affirm that $H_{0}$ corresponds to the combined effect of the Peierls stress and the work-hardening produced by an increase of dislocation density [50]. For pure $\alpha$-iron, the hardness related to the Peierls stress ( $H_{p} \approx 3 \sigma_{p}=334.2 \mathrm{MPa}$ ) [35] corresponds approximately to the material constant proposed in the model of Fu et al., ( $H_{o-F / Z}=300 \mathrm{MPa}$ ) [48]. It would mean that the discrepancy between both constants $\left(H_{0-A p r x}\right.$ and $\left.H_{0-F / Z}\right)$ is essentially related to the high strengthening level produced by severe plastic deformation during the shot-peening process. In Fig. 12 this corresponds to the "Dislocation Hardening" region, which represents the vertical rise of the straight blue line due to the increase of dislocation density. In other words, Fig. 12 shows the different effects involved in the material strengthening caused by the mechanical surface treatment and the micro-structural refinement. The first region $\left(H_{0}\right)$ represents the initial theoretical mechanical properties proposed in literature for a quasi-free dislocation $\alpha$-iron. The second zone (Dislocation Hardening) represents the increase of dislocation density due to severe plastic deformation. Note that the preexisting dislocation density state (or statistically stored dislocations, SSD) [51] of the material used in this work could also contribute to the hardness increase in this region. The last zone (Grain size effect) corresponds to the term $3 K_{h p} d^{-0.5}$ of the HallPetch equation. This zone is bounded by a grain size of $6 \mu \mathrm{m}$, from where the transition zone starts and there are no longer welldefined grains.
It is interesting to observe that the estimated value of $H_{0-A p r x}$ is between the bulk material constant measured with nano-indentation $\left(H_{0-N I} \approx 1250 \mathrm{MPa}\right)$ and the one quantified using the yield strength from micro-compression $\left(H_{0-M C} \approx 3 * 300 \mathrm{MPa}=900 \mathrm{MPa}\right)$ $[52,53]$. The discrepancy between these values may be explained by several reasons. Firstly, as was presented in the previous section, the hardness measurement corresponds to an averaged behavior of various slip systems, and the anisotropy perceived from nano-indentation testing is less significant. For that reason, some literature suggests that hardness measurements in single crystals can take higher values than those expected on the yield stress basis [36]. Secondly, the different crystal orientations of the single crystal zones where nano-mechanical testing was performed $\left(m_{\langle 111\rangle}=0.314 ; m_{\langle 225\rangle}=0.433\right)$ could enlarge the difference between both measurements. Moreover it could be possible that both nanomechanical test zones did not have the same increase in dislocation density after the mechanical surface treatment. As was previously observed, the dislocation density has a significant influence on mechanical property characterization. While not within the scope of this paper, quantification of this effect could be performed using high resolution EBSD, and will be the aim of future work.

The last question arising concerns possible residual stress effects on the measurement of mechanical property gradients, relating to the microstructural evolution in the cross-section. As shown by several authors $[54,55]$, residual stresses could confer a significant effect on nanoindentation results. However, the residual stress-free condition of the polycrystalline pillar and the good agreement between measurements in this region $\left(H / \sigma_{0.08} \approx 3.3\right.$ ) lead us to the conclusion that residual stress effects are effectively negligible when compared to microstructural factors. However, hardening induced by an increase in dislocation density can play a major role as was previously discussed. It is quite clear that addressing these two uncertainties could give more judicious interpretations for the determination of material constants $\left(H_{0}, K_{h p}\right)$ in pure $\alpha$-iron.

\section{Conclusions and perspectives}

In this work, an innovative approach based on the combination of two types of nano-mechanical tests was used to quantify the mechanical property gradient induced in pure $\alpha$-iron by an impact-based surface mechanical treatment. The grain size refinement produced in the near-surface and its influence on the hardness and yield strength evolution was additionally considered.

Both mechanical tests showed clearly an increase in mechanical properties due to the mechanical surface treatment. The nanoindentation results are generally consistent with the micro-compression results. The experimental Tabor's relationship between hardness and yield strength was confirmed, revealing results consistent with the expected ratio $\left(H / \sigma_{0.08}=3\right)$ classically used in literature [16,34], especially for tests carried out in polycrystalline regions.

The hardness gradient quantified by nano-indentation was closely related to the grain size distribution obtained from an EBSD map on the near-surface. The experimental data agrees with the theoretical Hall-Petch relationship, leading to the assessment of characteristic pure $\alpha$-iron constants $\left(H_{0}, K_{h p}\right)$. The grain size effect observed in this work is consistent with several model parameters proposed in literature for pure $\alpha$-iron $[48,49]$. Based on this analysis, it is obvious that grain size refinement has a considerable influence on the increase of mechanical properties and the evolution of the material's mechanical behavior, along with the presumably significant influence of dislocation density increments due to severe plastic deformation. Quantification of this effect will 
be the aim of future work.

Comparing both techniques, nano-indentation seems to be the less arduous method for the measurement of mechanical property gradients. However, data analysis and results are considerably dependent on the contact model used. On the other hand, microcompression offers a direct way to estimate the material's local constitutive behavior law. Nevertheless, considering the laborious micro-pillar fabrication process in the polycrystalline region and the good agreement between both techniques, nano-indentation remains an advantageous method to qualify mechanical property gradients.

Furthermore, this paper highlights that pure iron is an appropriate model material for obtaining a well-defined TTS region in order to compare both techniques. Likewise, such a material is favorable for SEM imaging and EBSD mapping, as well as displaying good homogeneity for FIB fabrication processes.

Future work will investigate the correlation between micropillar compression curves and the representative stress-strain values obtained from nano-indentation tests [16,56]. Quantification of the mechanical property gradients presented in transformed surfaces induced by other kinds of contact loading treatment, as for high pressure torsion (HPT) $[7,45]$ or micro-percussion tests $[57,58]$ will also be addressed.

\section{Acknowledgement}

This work is supported by the LABEX MANUTECH-SISE (ANR10-LABX-0075) of Universite de Lyon, within the program "Investissements d'Avenir" (ANR-11-IDEX-0007) operated by the French National Research Agency (ANR). The authors would like to thank G. Blanc, S. São-João and M. Mondon from Ecole des Mines de Saint-Etienne for sample preparation and microstructural characterization via SEM imaging and EBSD mapping. Thanks are also given to the CLYM (Center LYonnais de Microscopie, http:// clym.insa-lyon.fr) for the access to the FIB/SEM (Zeiss NVision 40) and to N. Blanchard, A. Descamps-Mandine, Th. Douillard, and B. Van De Moortèle for technical help. CLYM is supported by CNRS, le Grand Lyon, and le Conseil Régional Rhône-Alpes (FED4092 France). Finally, the authors thank Dr. James P. Best for his contribution on the revision of the manuscript and the writing corrections of the final document.

\section{References}

[1] N.R. Tao, Z.B. Wang, W.P. Tong, M.L. Sui, J. Lu, K. Lu, An investigation of surface nanocrystallization mechanism in Fe induced by surface mechanical attrition treatment, Acta Mater. 50 (2002) 4603-4616.

[2] K. Lu, J. Lu, Nanostructured surface layer on metallic materials induced by surface mechanical attrition treatment, Mater. Sci. Eng. A 375-377 (2004) $38-45$.

[3] H.W. Zhang, Z.K. Hei, G. Liu, J. Lu, K. Lu, Formation of nanostructured surface layer on AISI 304 stainless steel by means of surface mechanical attrition treatment, Acta Mater. 51 (2003) 1871-1881.

[4] H. Parvin, M. Kazeminezhad, Development a dislocation density based model considering the effect of stacking fault energy: severe plastic deformation, Comput. Mater. Sci. 95 (2014) 250-255.

[5] J. Aldazabal, J. Gil Sevillano, Hall-Petch behavior induced by plastic strain gradients, Mater. Sci. Eng. A 365 (2004) 186-190.

[6] V. Lacaille, G. Kermouche, D.Y. Tumbajoy Spinel, E. Feulvarch, C. Morel, J. M. Bergheau, Modeling nitriding enhancement resulting from the NanoPeening treatment of a Pure Iron, IOP Conf. Ser.: Mater. Sci. Eng. 63 (2014) 012124

[7] S. Descartes, M. Busquet, Y. Berthier, An attempt to produce ex situ TTS to understand their mechanical formation conditions - the case of an ultra high purity iron, Wear 271 (2011) 1833-1841.

[8] S. Descartes, C. Desrayaud, E. Niccolini, Y. Berthier, Presence and role of the third body in a wheel-rail contact, Wear 258 (2005) 1081-1090.

[9] G. Kermouche, J.L. Loubet, J.M. Bergheau, Extraction of stress-strain curves of elastic-viscoplastic solids using conical/pyramidal indentation testing with application to polymers, Mech. Mater. 40 (2008) 271-283.

[10] S. Schreijäg, D. Kaufmann, M. Wenk, O. Kraft, R. Mönig Size and microstructural effects in the mechanical response of $\alpha$-Fe and low alloyed steel, Acta Mater. 97 (2015) 94-104.

[11] Z. Sun, D. Retraint, B. Guelorget, L. Waltz, Micro-pillar compression tests to characterize the mechanical behavior of a nanocrystalline layer induced by SMAT in a 316L stainless steel, Matér. Tech. 103 (2015) 304.

[12] S.Q. Deng, A. Godfrey, W. Liu, N. Hansen, A gradient nanostructure generated in pure copper by platen friction sliding deformation, Scr. Mater. 117 (2016) $41-45$

[13] Xiaodan Zhang, Niels Hansen, Yukui Gao, Xiaoxu Huang, Hall-Petch and dislocation strengthening in graded nanostructured steel, Acta Mater. 60 (2012) 5933-5943.

[14] P. Zhang, S.X. Li, Z.F. Zhang, General relationship between strength and hardness, Mater. Sci. Eng. A 529 (2011) 62-73.

[15] I. Brooks, P. Lin, G. Palumbo, G.D. Hibbard, U. Erb, Analysis of hardness-tensile strength relationships for electroformed nanocrystalline materials, Mater. Sci. Eng. A 491 (2008) 412-419.

[16] M. Dao, N. Chollacoop, K.J. Van Vliet, T.A. Venkatesh, S. Suresh, Computational modeling of the forward and reverse problems in instrumented sharp indentation, Acta Mater. 49 (2001) 3899-3918.

[17] T. Prezeau, T. Muller, M. Baron, J. Samuel, E. Dransart, Surface treatment of a metal part, US Patent App. 14/126,602 (June 19, 2014).

[18] T. Prezeau, T. Müller, J. Samuel, Traitement de surface d'une pièce métallique par grenaillage oblique, EP Patent App. EP20,110,815,553 (November 6, 2013).

[19] Yu Ivanisenko, R.Z. Valiev, H.J. Fecht, Grain boundary statistics in nanostructured iron produced by high pressure torsion, Mater. Sci. Eng. A 390 (2005) 159-165.

[20] G. Guillonneau, G. Kermouche, J.M. Bergheau, J.L. Loubet, A new method to determine the true projected contact area using nanoindentation testing, $\mathrm{C}$. R. Mec. 343 (2015) 410-418.

[21] J.L. Loubet, M. Bauer, A. Tonck, S. Bec, B. Gauthier-Manuel, Nano-indentation with a surface force apparatus, NATO Adv. Study Inst. Ser. E (1993) 429-447.

[22] W.C. Oliver, G.M. Pharr, An improved technique for determining hardness and elastic-modulus using load and displacement sensing indentation experiments, J. Mater. Res. 7 (6) (1992) 1564-1583.

[23] J.L. Bucaille, S. Stauss, E. Felder, J. Michler, Determination of plastic properties of metals by instrumented indentation using different sharp indenters, Acta Mater. 51 (2003) 1663-1678.

[24] J. Woirgard, J.C. Dargenton, C. Tromas, V. Audurier, A new technology for nanohardness measurements: principle and applications, Surf. Coat. Technol. 100-101 (1998) 103-109.

[25] P. Juran, P.J. Liotier, C. Maurice, F. Valiorgue, G. Kermouche, Investigation of indentation-, impact- and scratch-induced mechanically affected zones in a copper single crystal, C.R. Mec. 343 (2015) 344-353.

[26] J.Y. Zhang, G. Liu, J. Sun, Strain rate effects on the mechanical response in multi- and single-crystalline Cu micropillars: grain boundary effects, Int. J. Plast. 50 (2013) 1-17.

[27] M. Uchic, D.M. Dimiduk, A methodology to investigate size scale effects in crystalline plasticity using uniaxial compression testing, Mater. Sci. Eng. A 400-401 (2005) 268-278.

[28] E.M. Grieveson, D.E.J. Armstrong, S. Xu, S.G. Roberts, Compression of self-ion implanted iron micropillars, J. Nucl. Mater. 430 (2012) 119-124.

[29] R. Rabe, J.M. Breguet, P. Schwaller, S. Stauss, F.J. Haug, J. Patscheider, J. Michler, Observation of fracture and plastic deformation during indentation and scratching inside the scanning electron microscope, Thin Solid Films 469-470 (2004) 206-213.

[30] G.E. Fougere, L. Riester, M. Ferber, J.R. Weertman, R.W. Siegel, Young's modulus of nanocrystalline Fe measured by nanoindentation, Mater. Sci. Eng. A 204 (1995) 1-6.

[31] J.I. Rojas, D. Crespo, Modeling of the effect of temperature, frequency, and phase transformations on the viscoelastic properties of AA 7075-T6 and AA 2024-T3 aluminum alloys, Metall. Mater. Trans. A 43 (2012) 4633-4646.

[32] D. Esqué-de los Ojos, J. Očenášek, J. Alcalá, Sharp indentation crystal plasticity finite element simulations: assessment of crystallographic anisotropy effects on the mechanical response of thin fcc single crystalline films, Comput. Mater. Sci. 86 (2014) 186-192.

[33] B.R. Sørås Rogne, C. Thaulow, Effect of crystal orientation on the strengthening of iron micro pillars, Mater. Sci. Eng. A 621 (2015) 133-142.

[34] G. Kermouche, J.L. Loubet, J.M. Bergheau, An approximate solution to the problem of cone or wedge indentation of elastoplastic solids, C. R. Mec. 333 (2005) 389-395.

[35] K.T. Kashyap, A. Bhat, P.G. Koppad, K.B. Puneeth, On peierls nabarro stress in iron, Comput. Mater. Sci. 56 (2012) 172-173.

[36] J.J. Vlassak, W.D. Nix, Measuring the elastic properties of anisotropic materials by means of indentation experiments, J. Mech. Phys. Solids 42 (8) (1994) $1223-1245$.

[37] O. Casals, S. Forest, Finite element crystal plasticity analysis of spherical in dentation in bulk single crystals and coatings, Comput. Mater. Sci. 45 (2009) 774-782.

[38] Y. Champion, Competing regimes of rate dependent plastic flow in ultra fine grained metals, Mater. Sci. Eng. A 560 (2013) 315-320.

[39] D. Kuhlmann-Wilsdorf, Theory of plastic deformation: properties of low energy dislocation structures, Mater. Sci. Eng. A 113 (1989) 1-41.

[40] J.Y. Zhang, S. Lei, Y. Liu, J.J. Niu, Y. Chen, G. Liu, X. Zhang, J. Sun, Length scaledependent deformation behavior of nanolayered $\mathrm{Cu} / \mathrm{Zr}$ micropillars, Acta 
Mater. 60 (2012) 1610-1622.

[41] C.P. Frick, B.G. Clark, S. Orso, A.S. Schneider, E. Arzt, Size effect on strength and strain hardening of small-scale [ $\left.\begin{array}{lll}1 & 1 & 1\end{array}\right]$ nickel compression pillars, Mater. Sci. Eng. A 489 (2008) 319-329.

[42] J.R. Greer, W.C. Oliver, W.D. Nix, Size dependence of mechanical properties of gold at the micron scale in the absence of strain gradients, Acta Mater. 53 (2005) 1821-1830.

[43] A. Abbasi, A. Dick, T. Hickel, J. Neugebauer, First-principles investigation of the effect of carbon on the stacking fault energy of Fe-C alloys, Acta Mater. 59 (2011) 3041-3048.

[44] T. Sakai, A. Belyakov, R. Kaibyshev, H. Miura, J.J. Jonas, Dynamic and postdynamic recrystallization under hot, cold and severe plastic deformation conditions, Prog. Mater. Sci. 60 (2014) 130-207.

[45] S. Descartes, C. Desrayaud, E.F. Rauch, Inhomogeneous microstructural evolution of pure iron during high-pressure torsion, Mater. Sci. Eng. A 528 (2011) 3666-3675.

[46] S.O. Gashti, A. Fattah-alhosseini, Y. Mazaheri, M.K. Keshavarz, Effects of grain size and dislocation density on strain hardening behavior of ultrafine grained AA1050 processed by accumulative roll bonding, J. Alloy. Compd. 658 (2016) 854-861.

[47] J.S.C. Jang, C.C. Koch, The Hall-Petch relationship in nanocrystalline iron produced by ball milling, Scr. Metall. Mater. 24 (1990) 1599-1604.

[48] H.H. Fu, D.J. Benson, M.A. Meyers, Analytical and computational description of effect of grain size on yield stress of metals, Acta Mater. 49 (2001) 2567-2582.

[49] M. Zhao, J.C. Li, Q. Jiang, Hall-Petch relationship in nanometer size range, J. Alloy. Compd. 361 (2003) 160-164.

[50] D.J. Dunstan, A.J. Bushby, Grain size dependence of the strength of metals: the
Hall-Petch effect does not scale as the inverse square root of grain size, Int. J. Plast. 53 (2014) 56-65.

[51] M. Zamanzade, J.R. Velayarce, O. Torrents-Abad, C. Motz, A. Barnoush, Mechanical behavior of iron aluminides: a comparison of nanoindentation, compression and bending of micropillars, Mater. Sci. Eng. A 652 (2016) 370-376.

[52] K.A. Padmanabhan, G.P. Dinda, H. Hahn, H. Gleiter, Inverse Hall-Petch effect and grain boundary sliding controlled flow in nanocrystalline materials, Mater. Sci. Eng. A 452-453 (2007) 462-468.

[53] J. Alcalá, D. Esqué-de los Ojos, Toward the development of continuum singlecrystal contact mechanics analyses to microindentation experiments, Comput. Mater. Sci. 52 (2012) 14-19.

[54] E. Frutos, M. Multigner, J.L. González-Carrasco, Novel approaches to determining residual stresses by ultramicroindentation techniques: application to sandblasted austenitic stainless steel, Acta Mater. 58 (2010) 4191-4198.

[55] P. Mann, H.Y. Miao, A. Gariépy, M. Lévesque, R.R. Chromik, Residual stress near single shot peening impingements determined by nanoindentation and numerical simulations, J. Mater. Sci. 50 (2015) 2284-2297.

[56] G. Kermouche, A.L. Kaiser, P. Gilles, J.M. Bergheau, Combined numerical and experimental approach of the impact-sliding wear of a stainless steel in a nuclear reactor, Wear 263 (2007) 1551-1555.

[57] G. Kermouche, G. Pacquaut, C. Langlade, J.M. Bergheau, Investigation of mechanically attrited structures induced by repeated impacts on an AISI1045 steel, C. R. Méc. 339 (2011) 552-562.

[58] H. Al Baida, G. Kermouche, C. Langlade, R.R. Ambriz, Identifying the stressstrain curve of materials by microimpact testing. Application on pure copper pure iron, and aluminum alloy 6061-T651, J. Mater. Res. 30 (2015) 2222-2230. 